Pure \& Appl. Chem., Vo1. 56, No. 9, pp. 1191-1202, 1984. Printed in Great Britain.
$0033-4545 / 84 \$ 3.00+0.00$

\title{
ELECTRON TRANSFER INDUCED PHOTOFRAGMENTATION AS A ROUTE TO FREE RADICALS
}

David F. Eaton

E. I. du Pont de Nemours and Company, Central Research and Development Department, Experimental Station, Wilmington, Delaware 19898, USA

Contribution No. 3489

Abstract. This paper describes the use of electron transfer induced photofragmentation reactions for the generation of free radicals. Two general reactions (eqns. 1,2) are presented. Equation 1

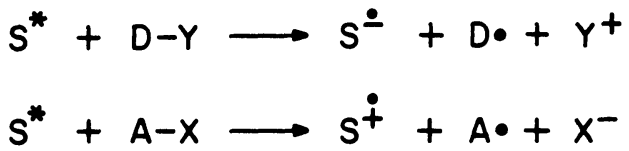

describes oxidative fragmentation of an electron rich species, D-Y, which quenches a sensitizer $S^{*}$ by donation of an electron to produce a kinetically unstable cation radical (D-Y+.) which rapidly dissociates to yield a radical (D.) and a cationic (Y+) fragment. Similarly, in eq. 2, reductive fragmentation results from quenching of $\mathrm{S}^{*}$ by an electron poor quencher $\mathrm{A}-\mathrm{X}$ whose anion radical (A-X-.) is kinetically unstable. Examples of each are presented, and the physical properties of the donor and acceptor which promote these processes are described.

\section{INTRODUCTION}

Organic photochemistry plays an important role in imaging technology. Photoinitiation of vinyl polymerization [1] is used reprographically to produce printed circuit boards, integrated circuits, printing plates and other graphic arts products, and in a variety of less well known imaging techniques [2]. Several of the primary photochemical processes useful in the formation of free radicals capable of intiating vinyl addition polymerization rely upon initial electron transfer between an excited sensitizer and an added substrate ("coinitiator")[3]. Following electron transfer, the resulting ion radical pair may suffer back electron transfer or proceed on to products. In the case of photoinitiation systems the desired products are often the initiating free radicals. Back electron transfer is a nonproductive step from the perspective of desired imaging chemistry, and is therefore to be avoided if possible. Such a competition between desired chemistry and back electron transfer is problematic in several photochemical applications, e.g. photovoltaic charge separation, photoelectrochemical energy storage schemes and other charge transfer systems [4]. Researchers continue to struggle with the important problem of avoiding charge recombination in these technologies. In photoimaging 
processes based on free radical polymerization we [5] have devised a simple method to avoid the complication of back electron transfer within charge transfer pairs: one (or both) of the ion radicals in the pair is specifically designed to be kinetically unstable with respect to fragmentation into an ion and a free radical. In this paper we describe two systems in which initial electron transfer is followed by rapid fragmentation of intermediate ion radicals.

\section{OXIDATIVE PHOTOFRAGMENTATION}

An electron rich species $D-Y$ which quenches an electron poor excited state $S^{*}$ by donation of an electron produces an ion radical pair, $\mathrm{S}-. /(\mathrm{D}-\mathrm{Y})+\ldots$ We define oxidative photofragmentation as the overall process which will result from such an electron transfer quenching if the intermediate cation radical can decompose into a cationic fragment $\left(\mathrm{Y}^{+}\right)$and a free radical (D.) in competition with back electron transfer. Scheme I summarizes the process.

\section{Scheme I}

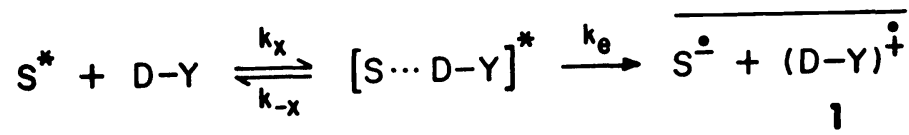

$$
\begin{aligned}
& \begin{array}{ll}
\stackrel{k_{-\theta}}{k_{f}} & S+D-Y \\
S^{\circ}+D \cdot+Y^{+}
\end{array}
\end{aligned}
$$

Examples of oxidative fragmentation were known prior to our work. Hammond [6] had described the exciplex interactions of the well-known photoinitiator combination [7] benzophenone-Michler's ketone. This system and other ketone/amine quenching pairs [8] function by proton transfer from the amine cation radical to the ketyl radical anion to produce a ketyl radical plus an alpha-amino radical. Ketone quenching by thiols [9] and quenching of aromatic excited states by disulfides [10] may also proceed by such a mechanism. Recently, Mariano [11] has employed such an oxidative fragmentation process in synthetic photochemistry. A host of dye sensitized electron transfer photoprocesses can be classed as oxidative fragmentations [3, 12]. Other electron transfer sensitized rearrangements [13, 14] and solvolyses [15] have also been described.

A key feature of all these systems is the use of a quencher possessing a low ionization potential. A second property which can serve as a useful indication of the likelihood that the intermediate cation radical will fragment is irreversibility of the electrochemical oxidation of the 
quencher. In the absence of this information some sense of the stability of the cation radical can be gained from the mass spectrum or the photoelectron spectrum of the quencher. Low abundance of the mass spectral parent ion or the observation of diffuse, featureless Franck-Condon envelopes for the lowest ionization bands of the photoelectron spectrum can be used to infer that the cation radical has a short lifetime.

All of these features obtain for organometallic compounds of the Group IVa metals ( $\mathrm{Si}, \mathrm{Sn}, \mathrm{Ge}$ and $\mathrm{Pb}$ ) [16]. We anticipated that facile exciplex mediated quenching of electron poor excited states would be exhibited by a variety of organometallic species and that electron transfer would be followed by rapid fragmentation of a carbon-metal bond to afford a cationic metal fragment and an organic free radical (Eq 4).

$$
R-M^{+} \longrightarrow R \cdot+M^{+}
$$

We chose to study organotin compounds initially because we expected that . they would be less toxic than germanium or lead compounds [17] and therefore safer to handle in thin film photopolymer compositions.

Since our interest was sensitization of photopolymerization to visible wavelengths, we employed photoreducible dyes [3, 18] as sensitizers. We prepared glassy thin film compositions consisting of a polar polymeric binder (e.g. the sodium salt of carboxymethyl cellulose or poly(vinyl alcohol)), a mixture of monofunctional and polyfunctional acrylate or methacrylate vinyl monomers, plasticizers and containing a dye (methylene blue (MB) or Rose Bengal (RB)) and benzyltrialkylstannanes 2 [5]. Exposure of these films to visible light (filtered tungsten source) resulted in imagewise polymerization of the monomers as evidenced by infrared spectroscopy (loss of the characteristic acrylate $810 \mathrm{~cm}^{-1}$ band), insolubilization of the imaged area (washout development) to form three

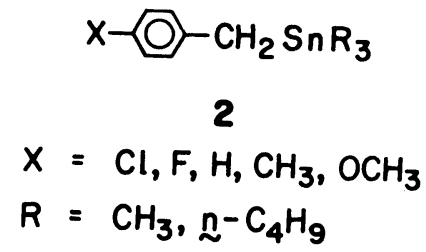

dimensional relief images and by direct precipitation and analysis of the polymer in model (solution) compositions. Dye photobleaching accompanied polymerization. We consider the observation of photopolymerization under the conditions described as direct evidence for the electron transfer quenching of MB excited state(s) by the organostannane and the subsequent fragmentation of the intermediate organotin cation radical since the semi-reduced form of MB (MB.) is known not to be capable of initiation [3, 5] but to suffer disproportionation to produce leuco-MB or demethylated derivatives [19]. Steady state kinetic studies show that the organotin derivatives quench the fluorescence of RB at near diffusion controlled rates $\left(\mathrm{K}_{\mathrm{SV}}=9.8 \mathrm{M}^{-1}\right.$ for $\operatorname{tri}(\mathbf{n}$-butyl)benzylstannane quenching in ethanol), 
and that the rate of photobleaching of $M B$ by the same organotin derivative (at constant concentration) depends on the square root of the incident light intensity $(655 \mathrm{~nm})$. These data imply that efficient excited state quenching by the organotin leads directly to photobleaching.

While we felt that the photopolymerization results were

compelling evidence for oxidative fragmentation as a result of bimolecular quenching in this system, we desired more fundamental physical organic insight into the processes. We therefore investigated the photophysics and photochemistry of the bimolecular interactions of organotin compounds and electron deficient anthracene singlet states [20]. Our studies, summarized below, support an overall reaction sequence as indicated in Scheme II in which $\mathrm{S}$ represents an anthracene

\section{Scheme II}

$$
\begin{aligned}
& S \stackrel{h \nu}{\longrightarrow} S^{*} \\
& S^{*}+B-T \underset{k_{-c}}{\stackrel{k_{e}}{\rightleftharpoons}}[S \cdots B-T]^{*} \\
& {[S \cdots B-T]^{*} \stackrel{k_{r} \longrightarrow}{\stackrel{k_{p}}{\longrightarrow} \text { Product } 3+B-T}}
\end{aligned}
$$

(5)

sensitizer, B-T a benzyltin quencher (2), and the product (cf structure 3 ) is formed by oxidative addition of the $B$ and $T$ fragments of 2 ( $B=$ benzyl, $\mathrm{T}=$ trialkyltin) across the anthracene nucleus. Fluorescent anthracene singlets with excited state redox potentials between 1.57 and 2.57 volts $\left(E^{*}{ }_{1 / 2}\right.$ VS SCE) [21] were quenched by a series of substituted benzylic trialkylstannanes. Rate constants for quenching $\left(k_{q}^{f}=k_{q}^{p}+k_{q}^{r}=k_{e}\left(k_{p}+\right.\right.$ $\left.k_{r}\right) /\left(k_{-e}+k_{p}+k_{r}\right)$, see Table I) correlated with $E_{1 / 2}^{*}$ for constant quencher and with the ionization potential of the organotin compounds for constant fluoror. A Hammett treatment of the quenching data $\left(\log k_{q}^{f} v s \sigma\right)$ for the substituted benzyltin compounds 2 indicated that positive charge accumulated at the benzyl carbon during quenching. For the strongly oxidizing excited singlet state of dicyanoanthracene $\left(E^{*}{ }_{1 / 2}=2.57 \mathrm{v}\right)$, quenching rate constants for benzyltrimethylstannane were diffusion controlled in all solvents regardless of dielectric constant (from hexane to acetonitrile), while for the less powerful oxidant represented by the excited singlet of $9,10-d i c h l o r o a n t h r a c e n e ~\left(E^{*}{ }_{1 / 2}=1.95 \mathrm{v}\right)$ rate constants increased as solvent polarity increased (50x increase in $k_{q}^{f}$ for a $18 x$ change in dielectric constant). All of these correlations support the notion that extensive charge transfer accompanies the quenching event. 
The bimolecular interaction of 9,10-dicyanoanthracene with tri(n-butyl)benzylstannane results in photochemistry. Irreversible photobleaching of the anthracene chromophore occurs and products are formed which are consistent with the formation of the 9,10-adduct 3 which is the result of oxidative fragmentation of intermediate benzyl tin cation

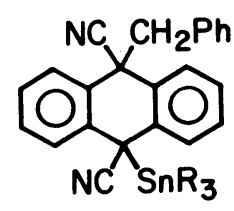

3

radicals and their cycloaddition to the anthracene anion radical. Steady. state analysis of the photobleaching kinetics implies that the product results from the exciplex. Rate constants for the product forming $s$ tep ( $\left.k_{p}^{q}\right)$ can be derived from the analysis and they can be shown to be independent of the nature of the para-substituent for the series of benzyl tin compound examined. However, efficiency of product formation (limiting quantum yield, $\left.\phi_{p}\right)$ is strongly dependent on the nature of the substituent, increasing as the substituent becomes more electron withdrawing. Table I summarizes all data derived from the analysis [20]. The results imply that

TABLE I. Rate Constants for Exciplex Decay Processes During Quenching of DCA by $\mathrm{p}-\mathrm{XPhCH}_{2} \mathrm{Sn}\left(\mathrm{CH}_{3}\right)_{3} \cdot \mathrm{a}, \mathrm{b}$

$\begin{array}{ccccc}\mathrm{x} & \mathrm{k} & \phi & \mathrm{f} & \mathrm{k} \\ \mathrm{Cl} & 3.8 & 0.91 & \mathrm{q} & \mathrm{k}_{\mathrm{q}}^{\mathrm{p}} \\ \mathrm{F} & 5.9 & 0.55 & 3.5 & 0.3 \\ \mathrm{H} & 7.9 & 0.45 & 3.2 & 3.1 \\ \mathrm{CH}_{3} & 10 & 0.25 & 2.5 & 4.8 \\ \mathrm{CH}_{3} \mathrm{O} & 21 & 0.11 & 2.1 & 7.5 \\ & & & \end{array}$

a. See Scheme II. Details in Ref. 20

b. All rate constants in $10^{9} \mathrm{M}^{-1} \mathrm{sec}^{-1}$.

non-radiative decay to ground states is rapid and in direct competition with product formation. We conclude that this non-radiative step is controlled by back electron transfer within the exciplex and that it is competitive with $\mathrm{C}-\mathrm{Sn}$ bond scission of the cation radical. We believe that this analysis is the most detailed view of the electronic factors which govern an exciplex mediated photochemical reaction acheived to date, in the absence of direct transient spectroscopic rate parameters.

\section{REDUCTIVE PHOTOFRAGMENTATION}

An electron deficient species $A-X$ which quenches an electron $r i c h$ 
excited state $S^{*}$ by accepting an electron produces an ion radical pair, $\mathrm{S}+. /(\mathrm{A}-\mathrm{X})$.. We define reductive photofragmentation as the overall process which will result from such an electron transfer quenching if the intermediate anion radical can decompose into an anionic fragment (X-) and a free radical (A.) in competition with back electron transfer. Scheme III summarizes the process.

\section{Scheme III}

$$
\begin{aligned}
S^{*}+A-X \underset{k_{-x}}{\stackrel{k_{x}}{\rightleftharpoons}}[S \cdots A-X]^{*} \stackrel{k_{e}}{\longrightarrow} \overline{S^{i}+(A-X)^{\circ}} \\
\stackrel{k_{-\theta}}{\longrightarrow} S+A-X \\
\stackrel{k_{f}}{\longrightarrow} S^{i}+A \cdot+X^{-}
\end{aligned}
$$

Numerous examples of reductive fragmentation can be found in both ground and excited state chemistry. Perhaps the best known example is Kornblum's photo- (or thermally) initiated radical ion chain substitution process [22] in which the initiation process involves electron capture by, for example, a p-nitrobenzyl halide, followed by loss of halide ion. Neta has shown by pulse radiolysis that the lifetimes of such intermediate nitrobenzyl halide radical anions are short [23].

We have employed a series of p-nitrobenzyl compounds and other low reduction potential moieties containing appropriately positioned leaving groups as electron acceptors in a variety of systems. One practical system is a photoimaging composition which uses solid semiconductor pigment particles such as $\mathrm{TiO}_{2}$ and $\mathrm{CdS}$ as the light absorbing element and includes substrates which suffer reductive photofragmentation upon capture of the photogenerated electron in the semiconductor [24]. The free radical generated by dissociative electron capture is used to initiate vinyl addition polymerization. Interestingly, this system can be employed also in an oxidative photofragmentation mode. Added reducing agents can trap photogenerated holes on the semiconductor surface. If the reducing agent is capable of oxidative fragmentation, for example a tertiary amine with alpha hydrogens, free radicals can be produced as a result of hole capture. In fact, we have developed compositions in which both oxidative and reductive photofragmentations can occur coincidentally, by capture of both the photogenerated electron and hole, to produce two initiating free radicals per photon [24]. Some of the evidence supporting this contention is described below.

The redox properties of semiconductor pigment particles are related to their band structure [25]. The fundamental photophysics of these 
materials has important implications for the application of semiconductors in photography [26; 27 electrophotography [28], photoelectrochemistry and photovoltaics [4]. The photooxidizing properties of $\mathrm{TiO}_{2}$ and other pigments are well known [29; 30; 31] and are mechanistically involved in the deleterious process of paint "chalking" [32]. Similarly, the ability of $\mathrm{TiO}_{2}$ and other pigments to participate in photoreduction, for example reducing oxygen to hydrogen peroxide or proton to hydrogen, is intimately linked to the conduction band energy and characteristics of these n-type semiconductors.

In our work, we have systematically examined the photopolymerization efficiency of compositions employing semiconductor pigment initiators of known redox properties. For these compositions we have varied the relative energetics of the pigment conduction band level and acceptor reduction potential as well as the pigment valence band level and donor oxidation potential. We have thus established families of electron acceptors which are effective with some pigments but not with others because the energetics of the electron transfer processes are either favorable or unfavorable. A similar family can be developed for electron donors by considering the dispositions of the valence band positions of the pigments and the oxidation potentials of the donors. Schematically, the overall situation for effective electron/hole separation and trapping can be depicted as shown in Scheme IV. Excitation of the semiconductor

\section{Scheme IV}
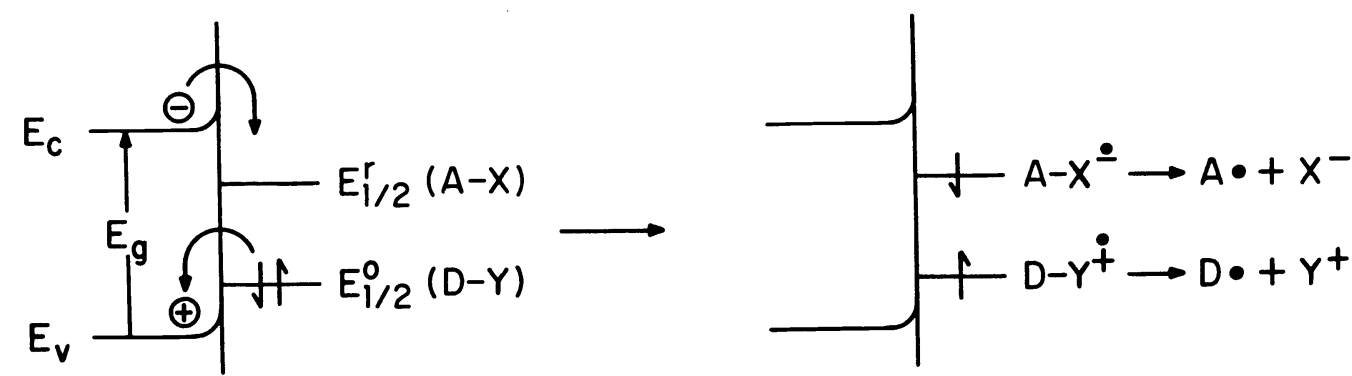

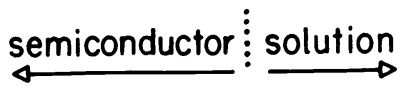

A

B

C

with light of energy greater than the band gap ( $\mathrm{F}_{\mathrm{g}}$ ) results in electron/ hole pair formation. Oxidizing agents $A-X$ whose redox potential is greater than $E_{C}$, the electrochemical equivalent of the conduction band edge at flat band conditions can trap the photoelectron to form ion radical A-X-. Similarly the oxidizing hole can be trapped by donors, D-Y with reducing power greater than $E_{v}$, the valence level potential. Part $A$ of Scheme IV 
illustrates the energetics required.

The situation just described is not sufficient, however to ensure photoinitiation, since electron/hole recombination can occur at any of several stages during the process. In fact, the reduction of the semioxidized donor $(\mathrm{D}-\mathrm{Y}+$.$) by the semi-reduced acceptor (\mathrm{A}-\mathrm{X}-.$.$) is clearly an$ exoergic process according to Scheme IV (cf part B). Such a process could be viewed as a form of surface state induced charge recombination, or, alternatively, as an electron transfer accelerated radiationless decay path. The charge recombination event might be retarded if the donor and acceptor moieties are spatially separated, either by chance or by specific adsorption to the pigment surface. A more effective means of preventing recombination would appear to be introduction of a kinetic instability to fragmentation in both ion radicals as shown in Scheme IV, part C. The overall process of photoinitiation can then be viewed as a pigment photoassisted photoredox reaction (Eq 12 ).

$$
\mathrm{A}-\mathrm{X}+\mathrm{B}-\mathrm{Y} \longrightarrow \mathrm{A} \cdot+\mathrm{B} \cdot+\mathrm{X}-+\mathrm{Y}+
$$

Evidence that both redox energetics and facility of fragmentation of the intermediate ion radicals is critical to the photoinitiation process is seen from the data in Table II. The Table lists normalized photospeeds of polymerizable compositions, under standard conditions. The compositions consist of (a) pigment, (b) electron acceptor, and electron donor (except for some control samples). Details of the preparation and characterization of the photopolymer films are found in ref. 24 .

The data show that for a given acceptor $\mathrm{A}-\mathrm{X}$, viable donor and pigment combinations are those for which $\mathrm{E}_{\mathrm{Ox}}^{\mathrm{l} / 2}<\mathrm{E}_{\mathrm{v}}$ (vs SCE). Similarly, efficacious acceptor/pigment pairs (for a constant donor) have $\mathrm{E}_{\text {red }}^{1 / 2}<\mathrm{E}_{\mathrm{C}}$. Additionally it is necessary, but not sufficient, that both conditions be satisfied simultaneously; i.e. $\mathrm{E}_{\mathrm{Ox}}^{1 / 2}<\mathrm{E}_{\mathrm{v}}$ and $\mathrm{E}_{\mathrm{red}}^{1 / 2}<\mathrm{E}_{\mathrm{C}}$. It is not sufficient to ensure polymerization since we also require that ion radical fragmentation must also occur. The data indicate that effective fragmentation for the series of p-nitrobenzyl derivatives 5

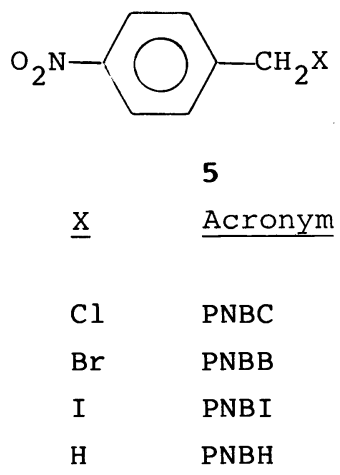


TABLE II. Relative Photopolymerization Efficiency of Pigment Semiconductor Compositions.

\begin{tabular}{|c|c|c|c|c|c|c|c|c|c|}
\hline Entry & Pigment $^{\mathrm{a}}$ & $\mathrm{E}_{\mathrm{g}}$ & $E_{v}-$ & $E_{C-}$ & $\mathrm{A}-\mathrm{x}^{\mathrm{b}}$ & $E_{r e d}^{1 / 2}$ & $\mathrm{D}-\mathrm{Y}^{\mathrm{C}}$ & $\mathrm{E}_{\mathrm{OX}}^{1 / 2}$ & $\begin{array}{l}\text { Rel. } \\
\text { Speed } \\
\end{array}$ \\
\hline 1 & $\mathrm{TiO}_{2}$ & 3.1 & 2.40 & -0.70 & PNBX & & & & \\
\hline a. & & & & & $\mathrm{X}=\mathrm{Br}$ & -1.1 & SPTS & +0.80 & 6 \\
\hline b. & & & & & $\mathrm{X}=\mathrm{Cl}$ & $"$ & $"$ & & 5 \\
\hline c. & & & & & $X=I$ & $"$ & $"$ & & 1 \\
\hline d. & & & & & $\mathrm{X}=\mathrm{H}$ & $"$ & $"$ & & 0 \\
\hline e. & & & & & ONBB & -1.1 & SPTS & & 0.7 \\
\hline f. & & & & & PBBB & -1.7 & SPTS & & 0 \\
\hline g. & & & & & PNBB & -1.1 & TEA & 1.0 & 12 \\
\hline $\mathrm{h}$. & & & & & PNBB & -1.1 & SCMC & 1.8 & 15 \\
\hline i. & & & & & PNBB & -1.1 & PVA & 2.3 & 10 \\
\hline $2 a$. & Zno & 3.1 & 1.85 & -1.15 & PNBB & $"$ & PVA & 2.3 & 0 \\
\hline $\mathrm{b}$. & & & & & $"$ & $"$ & TEA & 1.0 & 2 \\
\hline c. & & & & & $"$ & $"$ & SPTS & 0.80 & 4 \\
\hline $\mathrm{d}$ & & & & & - & - & $"$ & & 0 \\
\hline $3 a$. & $\mathrm{WO}_{3}$ & 2.8 & 1.90 & -0.80 & PNBB & -1.1 & - & - & 0 \\
\hline b. & & & & & PNBB & $"$ & TEA & 1.0 & 10 \\
\hline c. & & & & & $"$ & $"$ & SCMC & 1.8 & 2 \\
\hline $\mathrm{d}$ & & & & & $"$ & $"$ & PVA & 2.3 & 0 \\
\hline $4 a$. & $\mathrm{Cds}$ & 2.4 & 1.50 & 0.90 & PNBB & -1.1 & SPTS & 0.8 & 14 \\
\hline $\mathrm{b}$. & & & & & $"$ & & TEA & 1.0 & 16 \\
\hline c. & & & & & $"$ & & SCMC & 1.8 & 0 \\
\hline $\mathrm{d}$. & & & & & $"$ & & PVA & 2.3 & 0 \\
\hline $5 a$. & $\mathrm{Fe}_{2} \mathrm{O}_{3}$ & 1.9 & 1.5 & -0.4 & PNBB & -1.1 & SPTS & 0.8 & 0 \\
\hline
\end{tabular}

Notes: a. All redox values are in volts vs. SCE. Pigment redox values are adapted from M. S. Wrighton et al., J. Amer.

Chem. Soc., 99, 2839, (1977).

b. See structure 5 for acronyms for PNBX. Others: ONBB = o-nitrobenzyl bromide; $\mathrm{PBBB}=$ p-benzoylbenzyl bromide.

c. Acronyms: SPTS = sodium p-toluenesulfinate; $\mathrm{TEA}=$ triethanolamine; $\mathrm{SCMC}=$ sodium carboxymethly cellulose; $\mathrm{PVA}=$ poly(vinylalcohol).

d. See ref. 24 for details. 
(whose redox properties ensure facile electron trapping) is related roughly to the leaving group ability of the benzylic substituent $x$. Bad leaving groups (eg $\mathrm{X}=\mathrm{H}$, Entry ld) are not capable of generating radicals (no polymerization), but good leaving groups do efficiently induce polymerization $(\mathrm{X}=\mathrm{Br}, \mathrm{Cl}, \mathrm{I}$, Entries la-lc). However, the ability to initiate is not simply related to nucleofugality, since $\mathrm{X}=\mathrm{Cl}$ (Entry $1 \mathrm{~b}$ ) is more effective as an initiator than $\mathrm{X}=\mathrm{I}$ (Entry 1c), contrary to expectations based on leaving group ability rankings. Various explanations are feasible, but one probable cause is hole trapping by iodide ion to produce iodine atom. The resulting iodine could then recombine with nitrobenzyl radicals to regenerate starting material without initiating.

However, even use of a derivative of 5 which is capable of effective fragmentation, e.g. $5(x=B r)$, polymerization is not assured. An effective hole trapping agent must also be present. This principle is seen in Entry $3 a$ (no hole trap; no polymerization) in comparison to $3 b-d$ (for electron donors of varying redox potential). Compare especially $3 d$ which illustrates a donor (polyvinylalcohol, PVA) which is capable of hole trapping with the pigment $w_{3} \cdot$ Examples $4 \mathrm{c}$, d, vs $4 \mathrm{a}, \mathrm{b}$ illustrate this relation for Cds. These examples also show that in general, for a common pigment and electron acceptor, photospeed increases as the ease of oxidation of the added donor increases.

The data in Table II provide many more extensive examples of variations of electron donors than electron acceptors. We found only a limited number of effective acceptors. The nitrobenzyl derivatives 5 represent the only homologous series examined. Compounds containing leaving groups which reduce at more negative potentials vs SCE than 5 were inoperataive (e.g. Entry lf, p-benzoylbenzyl bromide PBBB). Even modest changes in the nitrobenzyl system are deleterious: o-nitrobenzyl bromide (Entry le) is only $1 / 10$ as effective as PNBB. other materials were tried. None were found to be effective.

The primary controlling parameters which define the efficacy of the systems are the redox levels of the pigments $\left(E_{v}, E_{c}\right.$ and the band gap $E_{g}$ ) and the $E_{1 / 2}$ values of the additives $A-X$ and $D-Y$. The failure of $\mathrm{Fe}_{2} \mathrm{O}_{3}$ (Entry $5 \mathrm{a}$ ) can be explained by the mismatch of energetics: the pigment photoelectron is incapable of reducing PNBB and the photohole cannot oxidize SPTS. It must be mentioned that these rules are generalizations, and not rigorous. Uncertainties in the redox potentials can be easily $\pm 0.2 \mathrm{v}$ for these materials.

Evidence has been obtained using ESCA [33], that the electron acceptor PNBB is adsorbed on the surface of $\mathrm{TiO}_{2}$ at approximately monolayer coverages. We find that the relative photopolymerization speed increases as the PNBB level is increased and then saturates as monolayer coverage is attained. Similarly, the ultimate photospeed which can be attained for a particular pigment-acceptor-donor combination is found to be higher for higher surface area pigments. In fact, if the pigment surface is treated with silicates to protect 
the native pigment surface and prevent adsorption of acceptor additives, then no photopolymerization is observed.

All the available evidence is consistent with the mechanism illustrated in Scheme IV in which photoproduced electrons and holes are trapped at the semiconductor surface by physi- (or chemi-) sorbed acceptors and donors. These trap sites react by bond fragmentation processes to yield radicals capable of initiation of polymerization.

\section{CONCLUSION}

We have described two examples of the use of photosensitized electron transfer chemistry to produce free radicals. The physical parameters which delineate the mechanisms of these photoreactions were illustrated for each of the examples. In both examples we used the photopolymerization of vinyl monomers as an analytical tool to rank the efficiency of comparative systems. This technique, if applied judiciously, can be a sensitive method to measure relative system quantum efficiencies for radical formation. The application of this technique in conjunction with conventional photophysical methods has been used to provide a detailed understanding of the mechanisms of electron transfer and radical ion fragmentation in these systems.

\section{REFERENCES}

1. L. Plambeck, US 2,760,863; "Photographic Preparation of Relief Images," issued August 28, 1956.

2. a. G. A. Delzenne, Adv. Photochem., 11, 1 (1975).

b. K. I. Jacobson and R. E. Jacobson, Imaging Systems, John Wiley \& Sons, Inc. N. Y. (1976).

C. E. Brinckman, G. Delzenne, A. Poot and J. Willems, Unconventional Imaging Processes, The Focal Press, London (1978).

3. G. Oster and N-L. Yang, Chem. Revs., 68, 125 (1968).

4. A. J. Bard, J. Phys. Chem., 86, 172 (1982).

5. D. F. Eaton, Phot. Sci. Engg., 23, 150 (1979); Proceedings, IUPAC Symposium on Photochemistry, Heverlee, July 1978, p. 124.

6. C. C. Wamser, G. S. Hammond, C. T. Chang, and C. Baylor, Jr., J. Amer. Chem. Soc., 92, 6362 (1970).

7. V. D. McGinniss, T. Provder, C. Kuo and A. Gallopo, Macromolecules, 11, 393, 405 (1978).

8. a. P. G. Stone and S. G. Cohen, J. Phys. Chem., 85, 1719 (1981).

b. S. Inbar, H. Linschitz and S. G. Cohen, J. Amer. Chem. Soc., 103, 1048 (1981).

C. J. D. Simon and K. S. Peters, Ibid., 103, 6403 (1981).

9. J. B. Guttenplan and S. G. Cohen, J. Amer. Chem. Soc., 94, 4040 (1972).

10. D. F. Eaton, unpublished.

11. P. S. Mariano, Tetrahedron, 39, 3845 (1984).

12. a. D. R. G. Brimage and R. S. Davidson, J. Chem. Soc., Perkin Trans. I, 496 (1973).

b. D. R. G. Brimage, R. S. Davidson and P. R. Steiner, Ibid, $526(1973)$.

C. R. S. Davidson, D. Goodwin and J. E. Pratt, Tetrahedron, 39, 2373(1983). 
13. S. L. Mattes, H. R. Luss and S. Farid, J. Phys. Chem., 87, 4779 (1983).

14. a. G. Jones, II, S.-H. Chiang, W. G. Becker and J. A. Welch, J. Phys. Chem., 86, 2086 (1982).

b. G. A. Lee and S. H. Israel, J. Org. Chem., 48, 4557 (1983).

15. H. F. Davis, P. K. Das, G. Griffin and J. D. Tampa, J. Org. Chem.' 48,5256 (1983).

16. a. R. S. Brown, D. F. Eaton, A. Hosomi, T. G. Traylor and J. M. Wright, J. Organomet. Chem., 66, 249 (1974).

b. A. Schweig, U. Weidner and G. Manuel, Ibid., 67, C4 (1974).

c. S. Fukuzumi, K. Mochida and J. K. Kochi, J. Amer. Chem. Soc., 101, 5961 (1979).

d. S. Fukuzumi, C. L. Wong and J. K. Kochi, Ibid., 102, 2928 (1980).

17. P. Smith and L. Smith, Chem. in Britain, 11, 208 (1975).

18. G. Oster, Nature (London), 173, 300 (1954).

19. a. R. B. McKay, Nature (London), 210, 296 (1966).

b. P. J. Hilson and R. B. McKay, Ibid., 210, 297 (1966).

c. F. C. Schaefer and W. D. Zimmermann, Ibid., 220, 66 (1968).

d. G. S. Singhal and E. Rabinowitch, J. Phys. Chem., 71, 3347 (1967).

20. D. F. Eaton, J. Amer. Chem. Soc., 102, 3280 (1980); Ibid., 103, 7235 (1981).

21. M. E. R. Marcondes, V. G. Toscano and R. G. Weiss, J. Amer. Chem. Soc., 97, 4485 (1975).

22. N. Kornblum, Angew. Chem., Int. Ed. Engl., 14, 734 (1975).

23. a. D. E. Bartak and M. D. Hawley, J. Amer. Chem. Soc., 94, 640 (1972).

b. P. Neta and D. Behar, Ibid., 102, 4798 (1980); J. Phys. Chem., 85, 690 (1981).

24. D. F. Eaton, U.S. 4,257,915, "Photopolymer Initiator System Containing a Semiconductor, A Reducing Agent, and An Oxidizing Agent," issued March 24, 1981; Presented at the 35th Annual SPSE Conference, Rochester, N. Y., May 9-14, 1982.

25. H. Gerischer and F. Willig, Top. Curr. Chem., 61, 31 (1976).

26. P. B. Gilman, Jr., Phot. Sci. Engg., 18, 475 (1974).

27. a. B. Levy and M. Lindsey, Ibid., 16, 389 (1972); 17, 135 (1973).

b. B. Levy, Ibid., 27, 204 (1983).

28. M. Pope and C. E. Swenberg, Electronic Processes in Organic Crystals, Oxford University Press, N. Y. (1982).

29. a. C. Renz, Helv. Chim. Acta. 4, 961 (1921).

b. R. I. Bickley and F. Stone, J. Catalysis, 31, 389 (1973).

c. F. Steinbach, Top. Curr. Chem. 25, 117 (1971).

30. B. Krautler and A. J. Bard, J. Amer. Chem. Soc., 99, 7729 (1977).

31. J. R. Harbour and M. L. Hair, J. Phys. Chem., 81, 1791 (1977); Ibid., 83, 652 (1979).

32. S. P. Pappas and R. M. Fischer, J. Paint Techn., 46, 65-72 (1974).

33. C. Batich and D. F. Eaton, unpublished. 